\title{
Analysis on the Interactive Mode in Network Media Environment
}

\author{
Yuming Sun \\ School of Arts \\ Northeast Dianli University \\ Jilin Province, Jilin City, China \\ E-mail: seusym@126.com
}

\author{
Xiaoling Fan \\ School of Arts \\ Northeast Dianli University \\ Jilin Province, Jilin City, China
}

\begin{abstract}
With the rapid development, Internet creates a digital network age, and establishes the digital survival mode. The transmission of information and the interaction within people are the basic motive force of social development, both of them are subject to the impact and influence from the Internet. Internet integrates the information from the traditional media with the digital processing technology, and reconstructs the information by non-linear Hypertext organization. Meanwhile, the continuous improvement and development of network communication also affects and changes the traditional mode of interaction.
\end{abstract}

\section{Keywords-internet; information; interactive mode}

\section{INTRODUCTION}

Internet as the important channel of information dissemination in today's society, is expanding rapidly in the global with its traits such as openness, virtual, interactive. Internet quickly penetrates into all areas of society, and changes the living environment, working way, even the ideas. United States media theorist Paul Levinson stated: "The computer-mediated communication and the advent of electronic interaction is very important, and is a significant achievement in the development of intelligent media. They have the similar status with the phonetic alphabet table and printing machine." With the development of computer and communication technology, the Internet grow up quickly, from the birth of prototype in the 1960s to the preparatory establishment in the 1980s. The Internet gradually extended to the business and entertainment areas from the applications of military, scientific and academic. The rapid development of Internet just like long-simmering storm swept through the global, brought the human society into the digital age with the core of information network. In the new media environment, the Internet will influence and change the traditional modes of information transfer, and establish the new types of interactive environments.

\section{The Evolution OF MEDIA AND the TRADITIONAL MODE OF INTERACTION}

Interaction is the most fundamental and universal phenomenon in everyday's life, and is the social reality we can experience directly. People come into being different social roles, establish social relations and form the social structures.
German sociologist Simmel said:"society is not the simple combination of people, there are also some modes of interaction, people can contact and influence each other via these patterns of interaction. Interactive is the action mode and process of mutual influence between the individual and individual, the individual and group, the group and group in society.

The interaction between humans is closely related with the development of the social productive forces and the media, the promotion of social productivity will result in new media, and the emergence of new media will change and affect the original mode of communication and interaction. [1]In primitive societies, speaking became the human's direct communication media after the body language. The appearance of speaking has greatly improved the quality, speed and efficiency of communication and exchange, enhanced the interaction and mutual cooperation between human, and promoted the progressive development of society.

However, the defects of speaking such as single, and shortdistance transmission, continued to hinder the human's communication and interaction. The invention of character allows people to save the oral information in symbolic form, and makes the oral information separate from speaker. The application of character overcomes the instability and unreliability of oral transmission in human's communication and interaction. The combination of character and typography makes the interaction become a dynamic process for using and cracking symbols, and develops the human imagination and abstract thinking. After entering the industrial society, telegraph, telephone, radio, television and other electronic communication technology and media have been invented, this broke down the space obstacle in some extent and expanded the scale of communication.

With the development of the media, the interaction between humans gradually got out the physical limitations and was extended with increasingly powerful information-carrying capacity of the media. Under the influence of mainstream media in different times, the transmission of human information went through the oral, written, electronic, and several major stages, and formed a parallel traditional patterns of interaction. 


\section{THE EXTERNAL FORMS OF NETWORK INTERACTION}

The rapid development of Internet, on the one hand, due to the improving of digital communication technology; On the other hand, the development power comes from the desire and effort of people in order to break through time and space barriers and achieve "barrier-free" communication and interaction. As two basic application at early stage, E-mail and BBS make the desire of exchange become reality, and provide the initial motivation for the development of Internet. [2]United Kingdom scholar John Norton says: "it can be said that the Internet is built on the basis of e-mail. Thanks to e-mail, global conferences and organizations, as well as media organizations expanded rapidly, APRA also gradually developed into today's Internet, in this process, email is undoubtedly the lubricants of network development.

Most amazing is that because the Internet is based on the human endless desire of interaction, therefore, it is the inexhaustible source of information." With the fast and convenient features, E-mail are replacing the traditional paper letters, and becoming the most frequently way of interactive. Email is the way of communication for one-to-one, and BBS is the main form of many persons interaction in the early stage. Large amounts of information are saved in BBS network servers, people can get information from the server, and also can upload and supplementary the information. E-mail and BBS realized the network communication and interaction between individual and individual, individual and group, and extended the narrow scope of interaction to more people from different geographical, partly replaced the traditional letters and face-to-face communication.

Although E-mail and BBS beyond the obstacle of physical space, from the perspective of time, they also belongs to the asynchronous interaction, not suitable for synchronous communication. As a rising star in the network, the instant messaging software can achieve synchronous exchange for online users. With the increasingly powerful function, the instant messaging software not only can send text, but also can transfer image and video. [3] The rise and maturity of instant communication software, has achieved and exceeded the communications functions of traditional media such as telegraph, telephone. The emergence of Web2.0 applications such as blogs, podcasts, have not only achieved the one to many communication and interaction, but also changing the knowledge system in network and social reality. The gradually development of the Internet affects communication and interaction of people, and participates the composition of social cultural.

\section{THE CONSTRUCTION OF INFORMATION ENVIRONMENT IN THE NETWORK INTERACTIVE}

The rapid rise of the Internet, is playing an increasingly important role in today's society, not only makes information transmission and communication more easily and quickly, and through the consolidation and reconstruction of the original transmission, is forming a new digital survival model. United States scholar Nicholas Negroponte believes that "bits" are taking the place of "atomic" becomes information "DNA" in the digital society. [4] Bits is the elementary particles of digital computation. More and more information, such as voice and video, have been digitized, and are reduced to the same 1 and 0 . In the 1990s, the emergence of Hypertext Transfer Protocol made the transmission of graphic information become possible. Since then, the interconnected network began to expand, from local to global, and formed a digital information exchange network system which gathered communications networks, computers, databases and other electronic products.

In this digital network systems, computers transmit data and information by the layered transmission protocol. "Network architecture from top to bottom includes the application layer, presentation layer, session layer, transport layer, network layer, data link layer and the physical layer. "In the Internet, the communication between computers directly show as service requests and feedback in the application layer. When a computer sends service request, it will convert to bit stream in physical line by following the different protocols, after arriving at the target computer, it will recover the information in the opposite order. When the results return from the target computer, it will go through the same process along the opposite path with the support of all levels of agreement. Therefore, the transmission of information such as text, image and audio in the Internet, actually is a digital conversion and transmission process of information within the network.

\section{A. The Digital Integration of Traditional Media Information}

With computers and the Internet as the representative, the digital communication technology provides a broad prospects for the development of the media. The notable different features between digital communication technology and traditional communication technology is the former can express different information content in the same carrier, and this feature provides a strong technical assurance for media convergence. Through digital processing technology, it can compress the "atomic" of traditional media into digital "bits", and make its encoding convert to "01" digital system. The digital technology can arrange the different content hosted by the traditional media into " 0 " and "1" order, and conduct unified storage, dissemination, and management of data.

The maturity and development of the digital processing technology not only can solve the problem of storage space in the process of information transmission, but also can easily convert the information in the traditional media to digital form, making it become a continuously source of Internet information. The digital media language of Internet, broke the boundaries of media, different media information achieve cross-media integration on this digital platform. [5] The information such as text, sound, image and video was used to perform and record the material and spiritual world of people, which can be digitized by computer binary language. The difference of traditional mass media such as books, newspapers, magazines, radio, television and film is gradually disappearing, when they merge with the Internet.

\section{B. The Digital Reconstruction of Traditional Media Information}

The Internet achieves the integration of traditional media information by the digital processing technology, and there is 
also a process of reconstruction in the network information transmission. United States scholar mark poster said: " just as printed, many forms of digital works separate authors from texts, but this also mobilize the text, so the reader can convert text by themselves, -- not only by his/her idea or some marginal notes, but add text itself change into another text to spread." The organization mode of network information has a lot of different with traditional media. In the traditional media, information is arranged in order, linear.

However, due to the hierarchical structure of information and the presence of hypertext links, the network information does not unfold in a closed unit, but presents the characteristics of Nonlinear and fragmentation. [6] The hyperlink presentation makes text become more open, multidimensional and divergence, which causes more connection with other information, so that every network text becomes a node in the whole information network. When people get information from the Internet, they often start from one node, but usually they will continue to jump to a new information node, it very fits the pattern of human thought, and increases the freedom of information received at the same time.

Human thought is inherently multidimensional divergence, the nonlinear organization form of network information much accords with human reading habits and thinking mode. The Internet integrates the traditional media information with digital processing technology, and reconstructs the information by nonlinear Hypertext.

\section{THE DEEP SIGNIFICANCE OF NETWORK INTERACTION}

Almost of human interaction initiates through a certain of medium. The face to face communication is just the main communication and interaction in early stage, but with the development of communication and information processing technology, the interaction between humans evolved a tendency, than is to break through limitation of time and space limitation by the medium function of transfer and continuation. United States media scholar Manuel Castells claims: "the new communication system in the Internet totally changes the basic dimension of human life: time and space. The regional disintegration separates from the significance of culture, history, geography, and reintegrates into the functional network or collage of images, leads to flow space replaces the local space." From the perspective of semiotics, the network interaction can be seemed as a dynamic exchange process of digital symbols. [7] The Internet interaction changes the traditional interaction mode of human-human, and creates a new style interaction of human-computer-human. In the human-computer interaction, the local interface instead of the remote computer, it brings the illusion to people that everything is in the local, makes people feel like interact with others who are pointed by the local computer interface.

When people used to a variety of computer interface, they will gradually forget the existence of interface, and pay attention to the communication process, enjoy the convenience brought by the across space and time interaction. In the Internet, people communicate between each other via interface, the interaction between people goes beyond the boundaries of present and absent. This digital network interactive also overcomes the limitations of the previous point to point communication, people are free to choose various forms of communication such as one-to-many, many-to-many and many-to-one.

Meanwhile, the virtual property of network, allows people to create an another "self" in the digital space which is different from the reality identity, and can maintain several different "self" in the one-to-many interaction. Although the network communication and interaction with virtual property, from the interactive process of human-computer-human, the network is just a new medium used by people to interact, and the interactive process is prompted only by the people in the social reality. [8]It is just because the Internet has the characteristics such as virtual and freedom, the network communication and interaction could become more convenient and quick, the Internet could become one of the most important information transmission channel in today's society, and gradually replaces the traditional interaction and communication.

\section{CONCLUSIONS}

The Internet is information dissemination platform based on digital technology, digital is the most essential attribute. With the powerful inclusiveness of digital technology, the Internet can combine the information of the traditional media such as books, newspapers, radio, television etc, and makes them become the important source of information. The material hosted form of information is converted to digital existence, the transmission of information in network shows as the flow of bits. Meanwhile, the nonlinear and fragmented properties of Internet, also changes the combinations of information. The Internet integrates the traditional media information with digital processing technology, and reconstructs the information by nonlinear Hypertext. The interactive mode based on traditional media is also affected by the Internet. In the digital network space, people communicate between each other via interface, the interaction between people goes beyond the boundaries of present and absent. With the further development of the Internet, the traditional way of communication and interaction will not only be replaced by the corresponding network form, but will also be transcended. The continuous improvement of the Internet technology and the increasing extension of transmission range, make the Internet gradually become the virtual space that parallel with the real society. The information transmission and communication is the basic guarantees of human society for normal operation, and maintain the development and progress of society. The rapid rise of the Internet not only influences the traditional information forms, but also changes the traditional mode of interaction.

\section{REFERENCES}

[1] Douglas Kenrick, Steven Neuberg, Robert Cialdini, Social Psychology: Goals in Interaction,5th ed.,2009, pp325-326.

[2] Johnny Ryan, A History of the Internet and the Digital Future, 2nd ed., Reaktion Books, 2013, pp67.

[3] Paul Levinson, New New Media, 2nd ed., Pearson Education, 2012, pp122.

[4] Nicholas Negroponte, Being Digital, 1st ed., Vintage,1996, pp16. 
[5] Mark Poster, Information Please: Culture and Politics in the Age of Digital Machines, 1st ed., Duke University Press, 2006, pp216.

[6] Jose van Dijck, The Culture of Connectivity: A Critical History of Social Media, 1st ed., Oxford University Press, 2013, pp55.

[7] Andrew Chadwick, Internet Politics: States, Citizens, and New Communication Technologies, Oxford University Press,1st ed., 2006, pp1136-137.

[8] Tessa Joseph Nicholas, Introduction to Digital Culture: Living and Thinking in an Information Age, Cognella Academic Publishing, 1st ed., 2012, pp96. 